\title{
Reciprocidad ética en las transferencias intergubernamentales: entre solidaridad e igualdad $^{* * *}$
}

\section{Ethical reciprocity on intergovernmental transfers: Between solidarity and equality}

\author{
SUMARIO
}

Introducción. I. Legitimación democrática y base axiológica constitucional. 1. Concepción axiológica de descentralización política. a. Libertad, igualdad y solidaridad. II. Constitución y solidaridad. 1. Solidaridad interterritorial y federalismo fiscal. III. Reciprocidad ética: entre igualdad y solidaridad. Conclusión.

\section{RESUMEN}

En el contexto histórico en que se desarrollan los sistemas descentralizados de estructuración de poder siempre hay la preocupación de atender la diversidad regional con unidad, acomodando las heterogeneidades territoriales en los más diversos arreglos jurídico-institucionales. Tales cuestiones ganan mayor destaque cuando se refieren a aspectos financieros, momento en el que

* Post-doctor en Derecho Político Comparado, Universidad Pompeu Fabra. Post-doctor en Derecho Constitucional Comparado, Universidad Complutense de Madrid. Doctor en Derecho del Estado, PUC/SP. Máster en Filosofía del Derecho y del Estado, PUC/sP. Máster en Derecho Tributario, PUC/SP. Licenciado en Derecho, Mackenzie/sP. Investigador visitante en el Instituto de Derecho Comparado de la Universidad Complutense de Madrid, en el Centro Interdipartimentale di Ricerca e di Formazione sul Diritto Pubblico Europeo e Comparato (DIPEC) de la Università degli Studi di Siena, en el Observatorio de la Evolución de las Instituciones de la Universidad Pompeu Fabra, en la Université Paris 1 Pantheon-La Sorbonne, en la Université Paris 10 OuestNanterre y en el Korean Institute of Southeast Asian Studies (KISEAS). Profesor visitante en la Universidad de Castilla-La Mancha (España), la Universidad Lomas de Zamorra (Argentina) y la Korea University (Corea). Profesor del programa de posgrado en Derecho de la Universidade Comunitária da Região de Chapecó (Brasil). Director del Center for European Strategic Research (CESR) (Italia). Contacto: ernani.contipelli@ cesr-eu.com

** Recibido el: 17 de junio de 2015, aprobado el: 12 de octubre de 2015.

Para citar el artículo: E. CONTIPELLI, Reciprocidad ética en las transferencias intergubernamentales: entre solidaridad e igualdad, Derecho del Estado n. ${ }^{\circ} 35$, Universidad Externado de Colombia, julio-diciembre de 2015, pp. 27-39. DOI: http://dx.doi.org/10.18601/01229893.n35.02 
entra en escena una serie de importantes cuestiones como la redistribución de riquezas operadas por el poder central, con el reparto de ingresos recaudados, la existencia de instrumentos de nivelación por medio de transferencias intergubernamentales, la debida imputación de los ingresos obtenidos con los costes del atendimiento de intereses regionales, entre otras temáticas, lo que necesariamente exige la investigación de las bases axiológico-constitucionales informadoras de los Estados descentralizados, que son pautadas, sobre todo, en las concepciones de solidaridad e igualdad. Así, búscase debatir la influencia de la reciprocidad ética, derivada de la idea de solidaridad, en el campo de las transferencias intergubernamentales. Para alcanzar ese objetivo se presentan los fundamentos teoréticos de la discusión centrada en la existencia de una base axiológica constitucional que se desarrolla en sintonía con la legitimación democrática de la experiencia jurídica, así como en el estudio del valor solidaridad, desvendando el núcleo de su contenido semántico, para dirigir las consideraciones en sus desdoblamientos interterritoriales, que lleva a la comprensión de la lealtad constitucional y la reciprocidad ética, las cuales justifican la atribución de deberes de colaboración en el desenrollar de las relaciones intergubernamentales, en especial, en términos financieros.

\section{PALABRAS CLAVE}

Descentralización política, Constitución, democracia, solidaridad, igualdad, reciprocidad ética.

\section{ABSTRACT}

In the historical context of the decentralized systems there is always the concern to attempt regional diversity with unity, settling territorial heterogeneities in diverse legal and institutional arrangements. Such issues gain greater prominence when referring to financial, which necessarily requires the investigation of the axiological-constitutional fundaments of decentralized states, which are based on the conceptions of solidarity and equality. The present article aims to discuss the influence of ethical reciprocity founded on the idea of solidarity in the field of intergovernmental transfers. To reach at this objective we will present the theoretical foundations of the discussion centered on the existence of a constitutional axiological basis, the democratic legitimacy of legal experience and the research of semantic profile of solidarity that acts directly at the inter-territorial issues to the understanding the ethical reciprocity, which justifies the attribution of collaboration duties on intergovernmental relations, especially relates to financial affairs. 
KEYWORDS

Decentralization Policy, Constitution, democracy, solidarity, equality, ethical reciprocity.

\section{INTRODUCCIÓN}

En el contexto histórico en que se desarrollan los sistemas descentralizados de estructuración de poder siempre está presente la preocupación de atender la diversidad regional con unidad, acomodando las heterogeneidades territoriales en los más diversos arreglos jurídico-institucionales, para posibilitar la cohesión y la convergencia entre la multiplicidad de intereses y una única propuesta de construcción y desarrollo nacional.

Tales cuestiones obtienen mayor relieve cuando se refieren a aspectos financieros, momento en el que entra en escena una serie de importantes cuestiones como la redistribución de riquezas operadas por el poder central, con el reparto de ingresos recaudados, la existencia de instrumentos de nivelación por medio de transferencias intergubernamentales, la debida imputación de los ingresos obtenidos con los costes del atendimiento de intereses regionales, entre otros, lo que necesariamente exige investigar las bases axiológico-constitucionales que informan los Estados descentralizados y que son pautadas, sobretodo, en las concepciones de solidaridad, libertad e igualdad.

Se busca por tanto debatir la influencia de la reciprocidad ética, que se deriva directamente de la idea de solidaridad interterritorial, en el campo de las transferencias intergubernamentales.

\section{LEGITIMACIÓN DEMOCRÁTICA Y BASE AXIOLÓGICA CONSTITUCIONAL}

La propuesta de concreción de los ideales contenidos en la base axiológica del Texto Constitucional demuestra los objetivos expresados en el proyecto de bien común que conduce a una sociedad, los cuales no pueden ser confundidos con posibles acciones realizadas por los poderes constituidos en el ámbito de las decisiones políticas, las cuales, corrompidas en determinadas ocasiones por intereses de carácter pragmático, no siempre se ajustan a los criterios de justicia consagrados por las disposiciones valorativas constitucionales en relación con las expresiones que se manifiestan cotidianamente en la vida de los ciudadanos.

En este contexto, debemos aprehender la Constitución como un sistema procedimental de ordenación social que compone la dinámica de las partes 
estructurales del Estado, así como las finalidades descritas en el proyecto de bien común, lo cual, al poseer alto contenido axiológico, encarna la raíz de un sentimiento grabado históricamente en la memoria del pueblo y que se desarrolla y se actualiza en la realidad concreta para atribuirles validez a las acciones políticas realizadas en el espacio público y colectivo. Surge así la idea de derechos y deberes como resultado de la base axiológica del proyecto constitucional de bien común.

En efecto, en el entorno social podemos encontrar diversos intereses manifestados por los ciudadanos y por los diferentes grupos sociales existentes, intereses que deben ser considerados jurídica y políticamente por los órganos y autoridades responsables de mantener el poder estatal en sintonía con el Texto Constitucional y la base axiológica contenida en su proyecto de bien común.

Para saber el sentido que debe ser atribuido a esa base axiológica, la función interpretativa desempeñada por los poderes constituidos juega un papel fundamental, ya que imprime las facciones del ideal de justicia, correspondiente con la realidad vivida socialmente, en el proceso de formación y actualización de las decisiones orientadas a atender las demandas sociales, lo que exige la participación de los agentes concernidos, propiciando para la estructura tutelar estatal un acercamiento a la verdadera concepción de "bien común" que es anhelada por la sociedad y que posibilita la legitimación democrática de su poder.

Por lo tanto, ser legítimo es ser fruto del acuerdo, de la aceptación de las partes incluidas en ese concepto. En el campo político, la legitimidad debe equilibrar los presupuestos abstractos de representatividad que posibilitan el funcionamiento automático del sistema de poder, con elementos concretos, que denotan las exigencias procedentes de la realidad social, lograda a partir de la adopción de procedimientos interpretativos adecuados, puede incrementar los atributos de legitimación democrática, confianza y eficacia social, necesarios para interpretar la base axiológica constitucional, y para aclarar sus respuestas a las pretensiones dictadas por el sentimiento público de justicia.

\section{Concepción axiológica de la descentralización política}

A partir de lo anteriormente mencionado se puede establecer que, con la apertura a un mayor contenido de legitimación democrática en el trato de los temas que rodean las singularidades propias de las unidades constitutivas, los sistemas estatales descentralizados, además de hacer valer su esencia plural, tienen más posibilidades de actuar con eficiencia en la resolución de conflictos eventuales entre unidades territoriales, realizando acciones que incentivan el robustecimiento de los vínculos asociativos mediante la construcción de un ambiente adecuado a sus condicionantes histórico-culturales. 
En el sentido contrario, el no atender las diferencias regionales que componen a los Estados descentralizados dificulta el equilibrio de las relaciones derivadas de la aspiración de unidad con diversidad, que caracteriza ese modelo estatal, perjudicando la construcción y el desarrollo de lazos asociativos territoriales, lo cual conduce a una crisis de funcionalidad fundada en una estructura artificial de poder, que culmina en la pérdida de autenticidad y en la desconfianza respecto de la validez del sistema. En tales términos, la propuesta de descentralización política dibujada constitucionalmente no alcanza cualidades mínimas de legitimidad jurídica y democrática y, por el contrario, presenta un grave déficit derivado de su absoluta falta de correspondencia con los factores empíricos y las diversidades sociales que aportaron en su formación y que están en un proceso constante de actualización social.

Tal como expone Ferdinand Lassale, el plan constitucional debe corresponder a una suma de factores reales de poder, para así no caracterizar a la Constitución solo como una "hoja de papel"1, que temprano o tarde sucumbe a las exigencias del mundo concreto y que por tanto exige una constante aproximación con las disposiciones jurídico-constitucionales positivadas.

Entendida bajo estas condiciones, la Constitución debe ser concebida en su aspecto procedimental, apta para absorber y alcanzar la multiplicidad de intereses de la experiencia social, disponiendo de reglas apropiadas para el desarrollo de los procesos de negociación y decisión juspolítica, independiente de elecciones realizadas por fórmulas preconcebidas e incluso de orientaciones ideológicas predeterminadas; lo anterior posibilita el enfrentamiento democrático entre distintas posiciones, abarcando todas las opiniones posibles, con el fin de alcanzar el consenso sobre la mejor manera de superar los problemas, incluso aquellos relacionados con los elementos informadores de la estructura territorial de poder y de acomodación de heterogeneidades regionales.

\section{a. Libertad, igualdad y solidaridad}

Al momento de analizar la relación entre valores y descentralización política debemos abarcar la aspiración de unidad con diversidad, ya que es relevante el considerar la experiencia social en la formación constitucional de un Estado y su correspondiente pacto asociativo territorial. Como respuesta a las condicionantes histórico-culturales, los modelos constitucionales de organización de poder intentan observar las diversidades regionales presentes en el territorio y crear las condiciones necesarias para articular tales diferencias, introduciendo en la base de la estructuración estatal los valores libertad, 
igualdad y solidaridad y preparando simultáneamente el terreno para fragmentar el poder, mantenerlo vinculado en determinadas dimensiones espaciales y actualizarlo, es decir, postular la adopción constitucional de un sistema de descentralización política del poder en línea con la dinámica de la vida social de toda nación y de sus ciudadanos.

Es por ello que la compatibilización entre las condicionantes axiológicas de la aspiración de unidad con diversidad va más allá de un juego de intereses políticos pragmáticos, involucrándose con valores básicos que actúan en la composición del proyecto constitucional de bien común que guía a los Estados descentralizados.

En otros términos, el valor de la libertad, como pilar de la descentralización política, delimita la autonomía de las unidades constitutivas, en el mantenimiento de sus singularidades, aproximándose a una concepción de existencia y preservación de las diversidades territoriales. Por su parte, la igualdad actúa sobre la distribución equitativa del poder y la participación en decisiones políticas respecto de intereses comunes entre las unidades constitutivas, con un despliegue amplio y homogéneo de servicios fundamentales para garantizar condiciones de vida satisfactoria y digna a todos los ciudadanos. Finalmente, la solidaridad surge como una propuesta axiológica de ayuda y respeto recíproco entre unidades constitutivas, lo que posibilita el desarrollo interterritorial equilibrado de los diversos espacios de manifestación (social, político, económico, entre otros) y en sintonía con la garantía de protección jurídica de ciertos rasgos histórico-culturales que contribuyeron en formación del pacto asociativo territorial, como lo es la presencia de minorías nacionales.

Desde esta perspectiva, la actualización de un modelo de descentralización político-constitucional debe garantizar una "fisonomía pluralista de poder" no pudiendo ser concebida como una simple inclinación orientada a la preservación de una alianza utópica, sino como una actitud concreta y dirigida constantemente a articular, con protección y respeto, el contenido de las diversidades regionales, de los intereses territoriales peculiares y del rol de derechos fundamentales de los ciudadanos, que hacen posible la coexistencia de diferencias y la representación en el ámbito público de todas las fuerzas políticas nacionales, en especial, de las minorías, y que consecuentemente aleja eventuales disposiciones al centralismo, al despotismo del grupo nacional hegemónico o incluso a un proceso de ruptura promovido por una minoría nacional que se sienta ignorada, para así mantener intacto y actualizado el pacto asociativo territorial. 
Resaltamos, así, la idea del respeto a las diversidades regionales, lo que permite la presencia de diferentes demandas en el seno de una nación, haciendo que sea necesaria la existencia de arreglos institucionales procedimentales que se inicien con la estructuración constitucional de un sistema fundado en la descentralización que se manifieste en instrumentos que promuevan la distribución de poderes ecuánimes y de carácter democrático, para la expresión de todas las voces presentes en la formación del vínculo asociativo territorial en las distintas esferas de gobierno con libertad, igualdad y solidaridad.

\section{CONSTITUCIÓN Y SOLIDARIDAD}

En su contenido semántico, la solidaridad relaciónase con el deber desarrollado en un ambiente de relaciones recíprocas, que alcanza a todos los segmentos de la sociedad y se orienta al apoyo conjunto de las situaciones de hiposuficiencias vividas por sus miembros, con el fin de asegurarles condiciones mínimas para su existencia digna y, recíprocamente, la correspondencia de comportamientos en los beneficiarios de estas acciones, quienes se esfuerzan para superar su situación de debilidad y así actuar también de manera altruista en favor del todo ${ }^{3}$.

Este relevante rol desempeñado por la solidaridad en el contexto social, como exigencia natural para la convivencia armónica, ha hecho que el valor no sea solo traducido como mera enunciación doctrinaria de la experiencia jurídica, agregando un plus de fuerza coercitiva estructurada en los modelos jurídicos, poseedores de estatus constitucional y de contenido abierto, lo que permite compatibilizar con la propuesta de interpretación procedimental para adaptarse con mayor flexibilidad a las constantes mutaciones de la realidad concreta y, de ese modo, promover la tutela correspondiente frente a las demandas, con eficacia social.

Como consecuencia, la preservación de esta apertura posibilita un amplio debate entre los diversos segmentos de la sociedad para identificar los focos de cooperación, de tal manera que su autorregulación sería retirada de las propias disposiciones procedimentales contenidas en el plan constitucional, siendo tarea del Estado el orientar adecuadamente el rumbo de estas actividades, en las reglas definidas en los preceptos normativos para su desarrollo, volviéndose así el centro de interacción de las "conciencias colectivas" "para formar las decisiones políticas fundamentales y la manifestación de la ciudadanía en 
conformidad con la realidad de las exigencias sociales y, consecuentemente, para compatibilizar las diversidades con la incidencia del valor solidaridad.

Esta perspectiva de análisis, basada en el sentimiento de solidaridad, otorga mayor eficacia social al fenómeno jurídico, acomodando y reduciendo las complejidades sociales, y atribuyendo un alto grado de confiabilidad al sistema, para una mayor aceptación de las decisiones políticas, establecidas por procedimientos abiertos y con la participación activa de los interesados.

Por tanto, la opción procedimental de la Constitución sugiere una aproximación continua y armónica de la pluralidad de valores existentes en el plan heterogéneo de la realidad social, teniendo como elemento nuclear la solidaridad, al permitir, por medio del compromiso entre los participantes del debate que presentan distintas opiniones políticas, y de la posibilidad de autorregulación, la compatibilización de disensos, de coexistencia entre diversa ideologías contrapuestas y, principalmente de una mayor consagración de los lazos de interdependencia entre los distintos grupos sociales, con una apertura en el canal de comunicación recíproco y con el reconocimiento del derecho de participación activa y de naturaleza eminentemente democrática.

\section{Solidaridad interterritorial y federalismo fiscal}

La solidaridad interterritorial alude a los lazos necesarios de interdependencia recíproca entre unidades constitutivas pertenecientes al modelo estatal descentralizado, que pasan a compartir derechos y deberes correlativos y subordinan sus actos políticos a la formación de beneficios espacialmente particularizados, y también dirigidos a los intereses generales de toda nación, los cuales están descritos en el proyecto constitucional de bien común.

Así, las unidades constitutivas de un sistema estatal descentralizado están sujetas a la realización de deberes de colaboración guiados por el ideal de cooperación recíproca e informado por el valor solidaridad, lo cual denota, en sintonía con el significado de este valor, la presencia de relaciones de auxilio y respeto mutuos, firmados en los vínculos de existencia común, que forman y participan del desarrollo empírico del pacto asociativo territorial que, concretamente, fundamenta la alianza nacional.

De ese modo, el panorama con que la solidaridad interterritorial se presenta frente a los Estados descentralizados implica la institucionalización de un conjunto de actos cooperativos pautados por una ética de reciprocidad entre unidades constitutivas que comparten la aspiración de unidad con diversidad, y donde se evidencia que cada unidad constitutiva se ponga en la posición de las demás, independientemente del contenido de las disparidades territoriales, para envolverse en la complejidad y particularidad de su vida política y de los consecuentes reflejos en el todo, en el cual están inmersas, reconocien- 
do la importancia de sus correspondientes papeles para la realización de las prescripciones solidarias a las que están vinculadas.

Importante resulta destacar que la solidaridad interterritorial se consagra, sobre todo, en la previsión de mecanismos de colaboración expresados por acciones convergentes entre los distintos niveles de gobierno presentes en un Estado políticamente descentralizado, existiendo así un régimen de distribución de competencias y beneficios compartidos que determina una especie de sistema abierto de comunicación entre las unidades constitutivas, para la expresión de decisiones destinadas a atender los más altos intereses de la colectividad, sean ellos nacionales, regionales o incluso locales, que se traducen en los objetivos fundamentales buscados por el conjunto de valores del Texto Constitucional, lo que lleva a la caracterización del denominado modelo estatal de descentralización cooperativa.

El reflejo de este modelo de cooperación surte efectos, principalmente, en la estructura de la organización financiera estatal a través de la consagración de mecanismos de transferencia intergubernamentales de nivelación, que, fundados en la existencia de heterogeneidades regionales que llevan a disparidades entre las unidades constitutivas, especialmente en cuanto a la variación de las posibilidades económicas y de capacidad fiscal ${ }^{5}$, evidencian la necesidad de reparto y redistribución de riquezas para propiciar el desarrollo nacional homogéneo, principalmente con la prestación de servicios públicos fundamentales de igual calidad en todo el territorio. Así, se tiene la idea de que los valores recaudados por ciertos tributos no son integralmente absorbidos por la entidad constitutiva competente para su institución, siendo compartidos con las demás.

En efecto, en el federalismo fiscal, la cooperación intergubernamental se expresa ante todo en la participación de los ingresos obtenidos con la recaudación, demostrando la existencia de una red de relaciones intergubernamentales orientada a la colaboración y prestación de auxilio mutuo en el territorio de una nación, pudiendo darse: de forma directa, a través de la participación de determinada unidad constitutiva en el producto de tributos recaudados por otra; o indirecta, por medio de la creación de fondos, en los cuales la cuota de ciertos tributos se destina a la formación de masa patrimonial que será afectada a una posterior distribución de sus valores entre unidades constitu-

5 La relación entre transferencias intergubernamentales (subvenciones) y diferentes capacidades fiscales como instrumento de ajuste es descrita por FRANCISCO GonZÁlEz BLANCH: "El deseo de la Hacienda central de compensar las diferentes posibilidades de las haciendas menores cuanto a la satisfacción de las necesidades públicas pueden servir para igualar la diferente posición de las distintas jurisdicciones cuando entre las mismas se detectan notables diferencias de "capacidad fiscal”" (GoNZÁlez BLANCH: 2008, p. 17). 
tivas beneficiarias, según criterios previamente estipulados que consideran, principalmente, la producción territorial de riqueza, para promover una mayor nivelación socioeconómica territorial.

\section{RECIPROCIDAD ÉTICA: ENTRE IGUALDAD Y SOLIDARIDAD}

En la formación y desarrollo de la "conciencia colectiva de existencia común" que permite evaluar los impactos concretos de tales actos cooperativos, fundados en la solidaridad, en lo referido a la constatación de efectos en la conjugación de ideales y acciones entre ciudadanos y entidades políticas, para hacer valer un sentimiento de pertenencia y construcción de nación, lo que demuestra la estrecha relación entre ese valor y la apertura procedimental-constitucional, que garantiza la participación democrática y activa de los agentes sociales en las decisiones políticas fundamentales que actúan sobre sus esferas de interés.

Justamente, en ese punto reside la importancia de analizar el contenido de la solidaridad, observado en el plan de las relaciones interterritoriales: el respeto para con el otro, que lo hace ser consciente del papel del otro en su propia composición y en la realización de los intereses generales de la nación. Vale decir que las diversas relaciones de reciprocidad entre unidades constitutivas que fundamentan derechos y deberes correlatos, existen y hacen parte de un todo, de tal modo que cada unidad constitutiva es parte integrante de las demás, que actúan conjuntamente para la realización del proyecto constitucional de existencia común.

No es exageración destacar una vez más, en este contexto, la acción del Texto Constitucional y su base axiológica, como documento jurídico estructural del modelo estatal descentralizado que posibilita la traducción institucional de los vínculos de solidaridad y de desarrollo de las consecuentes acciones convergentes entre unidades constitutivas para, desde el proceso de decisiones políticas fundamentales, posibilitar la continuidad del pacto asociativo territorial. La Constitución y sus valores se presentan como centro originario y congruente de la distribución de las esferas de poder político, con el reparto de competencias, visando a la debida manifestación de soberanía y permeando la disciplina de la forma de organización estatal con la descripción jurídica del modelo vigente.

Por lo tanto, las unidades constitutivas de un sistema estatal descentralizado, actuando sobre la base de una ética de reciprocidad que permea el contenido axiológico consagrado por las disposiciones constitucionales en el ejercicio de poderes y en la fruición de beneficios, se sitúan en la posición de las demás, reconociendo la dimensión de las diversidades regionales, parte de la experiencia histórico-cultural concreta, para permitir no solamente la formación del pacto asociativo territorial, sino también su actualización en términos evolutivos y compatibles con el proyecto constitucional de existencia común. 
Entonces, a través de la fuerza de la solidaridad interterritorial, las unidades constitutivas están sujetas a la realización de deberes de colaboración guiados por un ideal de cooperación ética recíproca, que demuestra la relación de respeto mutuo firmado en los vínculos de interdependencia y en la idea de "conciencia colectiva de existencia común" que influye la dinámica de un Estado descentralizado, para que así se responsabilicen por el cumplimiento de los encargos sociales, garantizando un nivel de dignidad igual en la vida y asegurando una calidad mínima de bienes esenciales para el pleno desarrollo de las libertades de decisión y de las potencialidades de los ciudadanos.

De ese modo, la idea de reciprocidad ética se refiere a la garantía de compromiso por parte de la unidad constitutiva beneficiaria que buscará una solución a las cuestiones que la llevaron a invocar los mecanismos de solidaridad, lo cual implica, por ejemplo, que la unidad constitutiva que recibe beneficios provenientes de las relaciones de cooperación basadas en la solidaridad, como un determinado ingreso proveniente de transferencias intergubernamentales, debe comprobar su compromiso ético con el pacto asociativo territorial y con el propio proyecto constitucional de bien común, demostrando que no solo merece la ventaja especial que le es conferida, sino que también empeña todo su esfuerzo político para la superación de la situación de adversidad. Este posicionamiento respecto de la reciprocidad ética en las relaciones de interdependencia que incluye la solidaridad, como se expuso anteriormente, se compatibiliza con su núcleo semántico.

Se trata de una simple correlación lógica vinculada al derecho de la unidad constitutiva que cumple con su deber solidario de colaboración, lo cual, atribuyendo racionalidad a la relación, consiste en la prestación de auxilio consciente de que la unidad constitutiva beneficiaria también se encuentra comprometida con la causa que fundamenta la realización de acciones convergentes dirigidas al proyecto de bien común y de desarrollo nacional basada en la orientación axiológica dada por la solidaridad.

De ese modo, la solidaridad interterritorial cumple un importante papel en el modelo de federalismo fiscal cooperativo, pasando a fundamentar la existencia de transferencias intergubernamentales de nivelación para crear un contexto de cooperación/colaboración financiera entre las unidades constitutivas, que abarca desde la idea del ejercicio de competencias firmadas y desempeñadas en el ideal de reciprocidad ética hacia el sentido de una vida satisfactoriamente digna a los ciudadanos.

\section{CONCLUSIÓN}

En la descentralización política, en que se pretende garantizar unidad con diversidad, el punto de equilibrio para establecer una relación de complementariedad 
entre autonomía y solidaridad en el desempeño de tareas constitucionales por las unidades constitutivas se hace necesario para permitir la efectiva manifestación de la aspiración de unión conciliada con el respeto a ciertas disparidades regionales que son parte integrante de las propias condicionantes histórico-culturales de estos modelos estatales de estructuración del poder.

Puesto en estos términos, la solidaridad se acerca a la idea de autonomía política y se sitúa en la relación de polaridad/complementariedad con la unidad, en la medida en que busca la protección de la esfera de autonomía de las unidades constitutivas prestadoras del auxilio (o también denominadas contribuyentes del sistema), que realizan esfuerzos para atender a sus intereses particulares, y que a través del auxilio de las demás buscan defender la causa mayor de ámbito general, siendo justo que al realizar este acto altruista se tengan las debidas garantías de que la unidad constitutiva beneficiaria también colabora en ese sentido.

Sin embargo, para propiciar el establecimiento de un ambiente fundado en la reciprocidad ética es necesario establecer una relación de correlación en la cual la solidaridad dialoga con la autonomía y la libertad, consagrándose el derecho de exigir la comprobación del compromiso de la unidad constitutiva beneficiaria con las propuestas del pacto asociativo territorial y del proyecto constitucional de bien común, demostrando su esfuerzo político en el sentido de superar la situación de hipo-suficiencia delante de la totalidad del sistema.

Es interesante observar que las relaciones de implicación recíproca entre los mencionados valores son inversas para las unidades constitutivas beneficiarias. Esto ya que la proximidad entre solidaridad/igualdad dirigida a la garantía de unidad se da con el deber de la beneficiaria de comprometerse ética y políticamente con la superación de su situación de adversidad; al paso que la solidaridad/libertad, orientada a la preservación de la autonomía, se realiza con el derecho de recibir auxilio para atender a los intereses territoriales fundamentales. Lo importante es que, con indiferencia del referencial adoptado (unidad constitutiva contribuyente o beneficiaria del sistema), hay un equilibrio en las relaciones de implicación recíproca envolviendo esos valores, que actúa en la preservación de los intereses de todos los concernidos por el pacto asociativo territorial.

Esta ecuación busca retratar la idea que se ha de tener de interdependencia ética recíproca y cooperación basadas en una solidaridad interterritorial que haga justicia a la aspiración de unidad con diversidad y que se pretende llevar a cabo en un Estado descentralizado, para que los mecanismos de colaboración no se concentren solo en el deber de auxilio recíproco de ciertas unidades constitutivas, sino también en el derecho de exigir que sus esfuerzos no sean en vano, que el sacrificio de la capacidad de atender a sus propios intereses territoriales tenga un sentido que se expresa en la comprobación de que todos 
los participantes del pacto asociativo nacional se encuentran debidamente comprometidos en su desarrollo.

En tales términos, se puede decir que la solidaridad interterritorial no puede ser para pocos, sino para todos, con el establecimiento de deberes de cooperación que busquen proveer a las unidades constitutivas en situaciones de contingencias con los instrumentos adecuados para la solución de problemas, y también con la previsión de derechos correlativos, que permitan a las unidades constitutivas que prestan auxilio la constatación de que no están "solas" en la construcción del proyecto conjunto de nación, lo que permite equilibrio suficiente en las relaciones entre los valores libertad e igualdad basadas en las directrices firmadas por la solidaridad, para alejar eventuales cuestionamientos y acciones políticas corrompedoras de los vínculos asociativos territoriales.

Por lo tanto, la solidaridad interterritorial exige que las unidades constitutivas ejerzan sus competencias teniendo en consideración el hecho de ser parte de un todo, con deberes y derechos correlativos, con intereses y necesidades coincidentes en los límites del dibujo institucional políticamente descentralizado con pauta en una propuesta ética de reciprocidad. Y además, que siendo parte de ese todo, respeten los intereses particulares de los demás, actuando no en contraposición, sino en complementación, sea como entidades realizadoras de deberes de colaboración o como entidades que se valen de los beneficios provenientes de arreglos solidarios.

\section{BIBLIOGRAFÍA}

Bidart CAmpos, Germán J. El poder. Buenos Aires: Ediar, 1995.

Contipelli, ERnANI. Solidariedade social tributária. Lisboa: Almedina, 2010.

Durkheim, Émile. Da divisão do trabalho social. São Paulo: Editora Martins Fontes, 2008.

GonzÁlez Blanch, Francisco. La Hacienda Pública descentralizada: teoría y evidencia empírica. Madrid: Universidad Complutense de Madrid, 2000.

Lassale, Ferdinand. A essência da Constituição. Rio de Janeiro: Lumen Juris, 2001. 